\title{
Clinicopathological characteristics and survival of primary malignant melanoma of the esophagus
}

\author{
TADAYOSHI HASHIMOTO $^{1}$, TOMOKI MAKINO ${ }^{1}$, MAKOTO YAMASAKI $^{1}$, \\ KOJI TANAKA ${ }^{1}$, YASUHIRO MIYAZAKI ${ }^{1}$, TSUYOSHI TAKAHASHI ${ }^{1}$, \\ YUKINORI KUROKAWA ${ }^{1}$, MASAAKI MOTOORI ${ }^{2}$, YUTAKA KIMURA ${ }^{3}$, \\ KIYOKAZU NAKAJIMA ${ }^{1}$, EIICHI MORII ${ }^{4}$, MASAKI MORI ${ }^{5}$ and YUICHIRO DOKI ${ }^{1}$ \\ ${ }^{1}$ Department of Gastroenterological Surgery, Osaka University Graduate School of Medicine, Osaka 565-0871; \\ ${ }^{2}$ Department of Surgery, Osaka General Medical Center, Osaka 558-8558; ${ }^{3}$ Department of Surgery, \\ Kindai University Faculty of Medicine, Osaka 589-8511; ${ }^{4}$ Department of Pathology, \\ Graduate School of Medicine, Osaka University, Osaka 565-0871; ${ }^{5}$ Department of \\ Surgery and Science, Graduate School of Medical Sciences, \\ Kyushu University, Fukuoka 812-8582, Japan
}

Received January 22, 2019; Accepted May 13, 2019

DOI: $10.3892 / \mathrm{ol} .2019 .10519$

\begin{abstract}
Primary malignant melanoma of the esophagus (PMME) has been reported to be a rare and highly malignant disease, and to date a standard treatment strategy has not been established due to limited evidence. The aim of the present study was to investigate the clinicopathological characteristics of this extremely rare disease. A total of 6 out of 2,093 patients with PMME treated in our institution between 1995 and 2016 were retrospectively analyzed and their clinicopathological parameters including treatment course and long-term survival were investigated. The major clinicopathological characteristics of patients were that they were $>70$ years of age, male sex, dysphagia at first diagnosis, and macroscopic black protruding tumors located in the lower third of the thoracic esophagus. Four of the five patients receiving pretherapeutic endoscopic biopsy were correctly diagnosed with PMME, and two patients received preoperative treatment with ineffective histopathological responses. There were two unresectable cases, one was treated with an immune-checkpoint inhibitor and the other received palliative care. Three of the four patients receiving curative surgery developed hematogenous recurrence within two years of surgery and only one patient with pT1aN0M0 achieved long-term survival. The median overall survival of all six patients was 19.6 (6.4-40.5) months. Patients with stage I disease exhibited significantly more favorable prognoses
\end{abstract}

Correspondence to: Dr Tomoki Makino, Department of Gastroenterological Surgery, Osaka University Graduate School of Medicine, 2-2-E2 Yamadaoka, Suita, Osaka 565-0871, Japan

E-mail: tmakino@gesurg.med.osaka-u.ac.jp

Key words: esophageal cancer, immune-checkpoint inhibitor, primary malignant melanoma of the esophagus, prognostic factor than those with stage II-IV $(\mathrm{P}=0.025)$ and surgically-treated patients had significantly better prognoses than those who did not receive surgery $(\mathrm{P}=0.018)$. In conclusion, PMME was associated with highly malignant features and tended to develop hematogenous metastases even after radical resection. Early diagnosis appears to be important to cure this refractory disease.

\section{Introduction}

Primary malignant melanoma of the esophagus (PMME) is an extremely rare disease accounting for only 0.1 to $0.5 \%$ of all primary esophageal malignancies (1). PMME is reported to be a dismal prognosis and develops multiple metastases even in early stage disease (2). Early detection of PMME has recently increased due to the relatively high prevalence of medical examinations and advances in diagnostic technology (3). However, prognosis remains poor due to the high metastatic potential of this disease. There are only a few reports of single or several cases of PMME, and a standard treatment strategy has not yet been established due to its rarity and the absence of strong evidence (4,5). Previous multi-institutional retrospective study conducted by the Japan Esophageal Society reported that the standard treatment of esophageal malignant melanoma was radical surgery, but its indications and additional options including chemotherapy, chemoradiotherapy, and endocrine therapy required careful thinking, and further studies were warranted (6). In addition, immunotherapy including immuno-checkpoint inhibitors has been an additional novel therapy in recent years, and has attracted attention as a useful treatment for cutaneous malignant melanoma (7).

Additional studies of PMME treated with various options are needed to understand the characteristics of this disease and establish a standard treatment. The present study therefore aims to review the clinicopathological characteristics and analyze the survival of six patients diagnosed with PMME at 
our institution and to summarize previously reported cases of PMME.

\section{Patients and methods}

Patients. The present study was approved by the Institutional Review Board of Osaka University Hospital (approved project no. 18190), and it conformed to the provisions of the Declaration of Helsinki. Written informed consent was obtained from all individuals in the present study. Six of $2,093(0.29 \%)$ patients with esophageal cancer treated at our institution between January 1995 and December 2016 were diagnosed with PMME and retrospectively analyzed. We investigated the clinicopathological characteristics of these patients including clinical symptoms, demographics, tumor characteristics, treatment courses, chemosensitivity, and survival. All patients were staged based on the seventh edition of the TNM classification as established by the Union for International Cancer Control and the clinical response to preoperative treatment was evaluated based on the revised RECIST guideline (version 1.1) $(8,9)$. The histopathological response to preoperative treatment was evaluated by the percentage of residual tumor volume compared to the estimated tumor volume prior to preoperative treatment and categorized according to the Japanese Society for Esophageal Disease: Grade 0 (ineffective), grade 1a (slightly effective, with a residual tumor that covers more than two thirds of the tumor bed), grade $1 \mathrm{~b}$ (slightly effective, with a residual tumor that covers more than one third of the tumor bed), grade 2 (moderately effective, with a residual tumor that covers less than one third of the tumor bed), and grade 3 (markedly effective, with no residual tumor) (10-12).

Preoperative and surgical treatment. Preoperative chemotherapy consisting of three cycles of the DAV regimen, dacarbazine $100 \mathrm{mg} / \mathrm{m}^{2}$ (days 1-5), nimustine $50 \mathrm{mg} / \mathrm{m}^{2}$ (day 1), and vincristine $0.5 \mathrm{mg} / \mathrm{m}^{2}$ (day 1), was administered for patients with pathologically diagnosed cT1N2 PMME according to the standard regimen for cutaneous malignant melanoma (13). Preoperative radiation $(50.4 \mathrm{~Gy} / 28 \mathrm{Fr})$ with the DCF regimen consisting of docetaxel $30 \mathrm{mg} / \mathrm{m}^{2}$ (days 1 and 8), cisplatin $10 \mathrm{mg} / \mathrm{m}^{2}$ (days 1-5), and 5-FU $400 \mathrm{mg} / \mathrm{m}^{2} /$ day (days $1-5$ ) was performed for patients with cT4aN1 esophageal cancer pathologically diagnosed as SCC by endoscopic biopsy, based on the indications at our institution as previously reported $(14,15)$. Surgery for PMME was carried out according to our standard surgical treatment for thoracic esophageal cancer and consisted of a subtotal esophagectomy with two or three field lymphadenectomies, reconstruction of the gastric tube via the retrosternal or posterior mediastinal route, and anastomosis in the cervical area from the cervical incision, as previously described (16).

Immunohistochemical staining. Biopsy samples and resected specimens were fixed in $4 \%$ formaldehyde. $3.5-\mu \mathrm{m}$ thick sections were prepared from formalin-fixed, paraffin-embedded (FFPE) blocks and hematoxylin and eosin (H\&E) staining was performed. Antibodies used for immunohistochemical staining included anti-HMB45, anti-Melan A, anti-S100, and anti-cytokeratin (CK) AE1/AE3.
The label was developed by 3,3'-diaminobenzidine tetrahydrochloride (DAB). HMB45, Melan A, and CK AE1/AE3 expression was found in the cytoplasm and S-100 was localized to the nucleus. Brown tumor cell cytoplasm or nucleus staining was considered a positive result.

Statistical analysis. Data are presented as mean values plus standard deviations or median values plus regions. Overall survival (OS) was defined as the time from the date of the first medical examination to the date of death from any cause or the date of the last follow-up, and recurrence-free survival (RFS) was defined as the time from the date of surgery to the date of recurrence. OS was estimated by the Kaplan-Meier method, and significant differences between groups were tested with the log-rank test. $\mathrm{P}<0.05$ was considered to indicate a statistically significant difference. All statistical analyses were performed with the SPSS Statistics software program, version 22 (IBM Corp., Armonk, NY, USA).

\section{Results}

Clinical and tumor characteristics. The clinicopathological characteristics, treatment courses, and prognosis of all six patients are summarized in Table I. Images from endoscopic examinations, CT and PET-CT imaging, and resected specimens are shown in Fig. 1. The median age was $76(55-84)$ years, and $66.7 \%$ (4/6) of the patients were male. Three patients $(50.0 \%)$ had dysphagia and another three patients $(50.0 \%)$ were referred to us following the detection of an esophageal lesion during a physical checkup at another hospital. Tumors were located in the upper (16.7\%), middle $(16.7 \%)$ and lower $(66.7 \%)$ third of the thoracic esophagus as shown in Fig. 1. The median length of the tumors was $60 \mathrm{~mm}$ (range, 29-80), and endoscopic examination revealed a dark gray protruding lesion in four patients $(66.7 \%)$, a dark gray superficial lesion in one patient $(16.7 \%)$, and reddish protruding lesion in one patient $(16.7 \%)$. All patients received endoscopic biopsies before treatment with the exception of one case (16.7\%), due to physician concerns about bleeding and dissemination associated with the tumor lesion (patient 1). Of the patients who received endoscopic biopsy, four $(80.0 \%)$ were correctly diagnosed with PMME while one (20.0\%) was misdiagnosed with poorly differentiated squamous cell carcinoma (patient 4). Four of the five patients (80\%, patients 2,3,4, and 6) who received ${ }^{18} \mathrm{~F}$-fluorodeoxyglucose-positron emission tomography/computed tomography (F-FDG PET/CT) showed abnormal uptake in the primary tumor (maximum standardized uptake value $\left(\mathrm{SUV}_{\max }\right), 5.67,25.6,23.4$, and 15.8, respectively). All patients received TNM staging and were diagnosed with cT1/2/3/4: 3/0/2/1, cN0/1/2: 2/3/1, and stage I/II/III/IV: 2/0/3/1, respectively.

Treatment courses. One patient (patient 3) had distant metastasis in liver, muscle, and bone (cStage IV), and palliative therapy was indicated in consideration of his advanced age and poor performance status. One elderly patient over 80 years of age (patient 6) was treated by immunotherapy with the immune-checkpoint inhibitor nivolumab, and received two cycles of this regimen without any adverse events. Response evaluation revealed stable disease after two cycles of anti-PD-1 


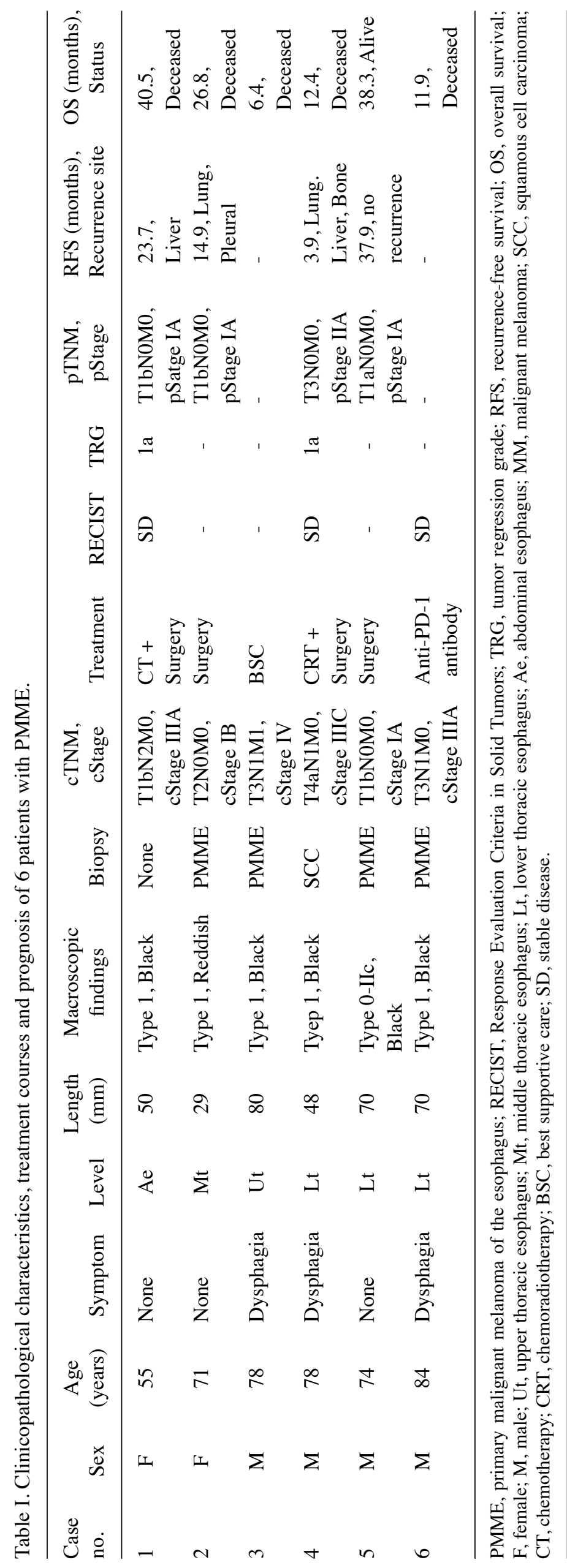



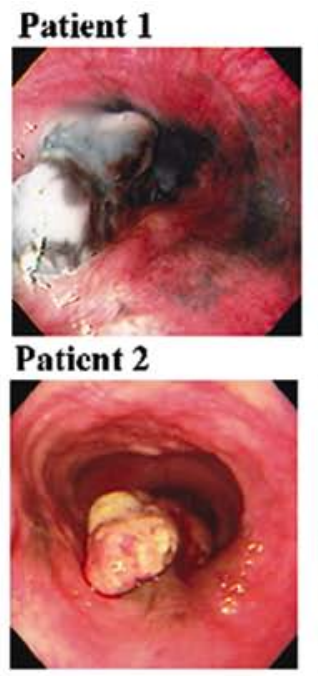

\section{Patient 3}

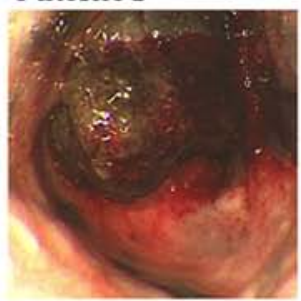

Patient 4

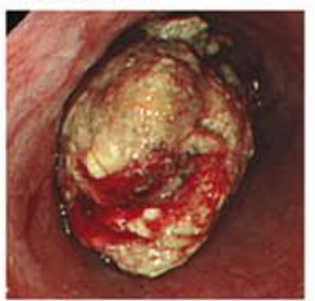

\section{Patient 5}

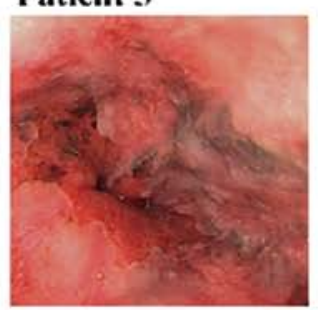

\section{Patient 6}

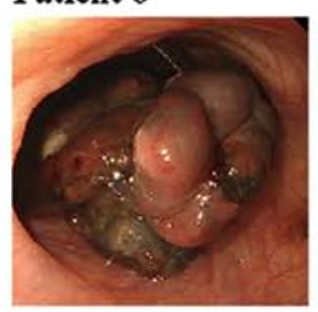

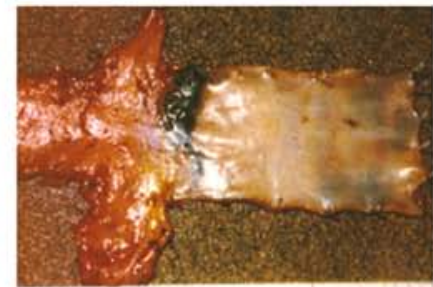
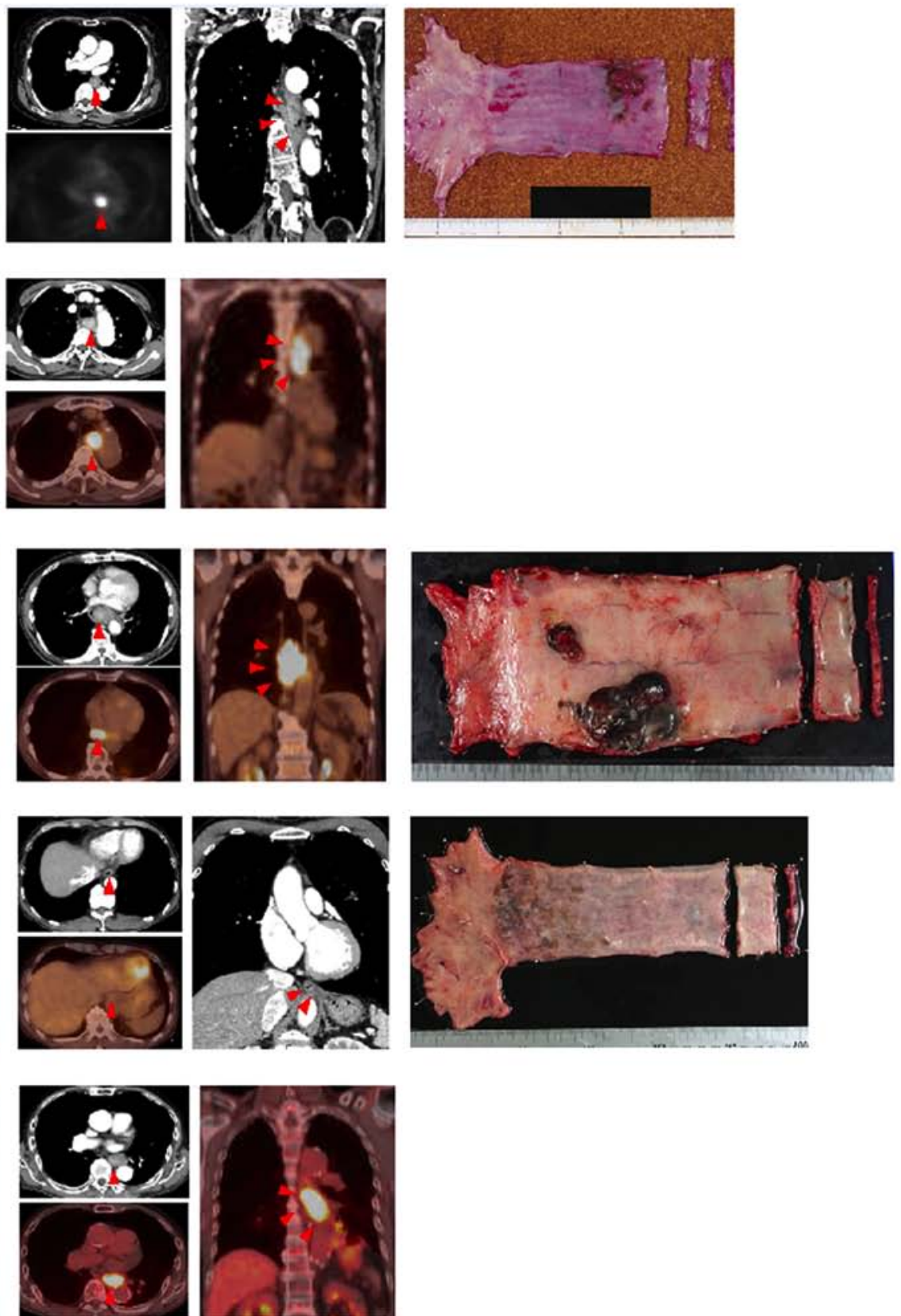

Figure 1. Representative examples of imaging results and resected specimens. Red arrowheads indicate tumors on CT and FDG-PET CT images. Patient 1 : (left-hand panel) endoscopy revealed a protruding-type tumor with a gray-black color; (right-hand panel) Resected specimen. Patient 2: (left-hand panel) endoscopy revealed a protruding-type tumor with a reddish color; (center panel) the top left and right panels show the CT images. The bottom left panel shows the FDG-PET CT images; SUV max, 5.67 abnormal FDG uptake in the tumor; (right-hand panel) Resected specimen. Patient 3: (left-hand panel) endoscopy revealed a protruding-type tumor with a gray-black color; (right-hand panel) the top left panel shows a CT image. The bottom left and right panels show FDG-PET CT images; SUV max, 25.6 abnormal FDG uptake in the tumor. Patient 4: (left-hand panel) endoscopy revealed a protruding-type tumor with a gray-black color, the surface of which was covered with necrotic epithelium; (center panel) the top left panel shows a CT image. The bottom left and right panels show FDG-PET CT images; SUV max, 23.4 abnormal FDG uptake in the tumor; (right-hand panel) Resected specimen. Patient 5: (left-hand panel) endoscopy revealed an ulcerative superficial-type tumor with a gray-black color; (center panel) the top left and right panels show CT images. The bottom left panel shows FDG-PET CT images; no abnormal FDG uptake in the tumor; (right-hand panel) resected specimen. Patient 6: (left-hand panel) endoscopy revealed a protruding-type tumor with a gray-black color; (right-hand panel) The upper left panel shows a CT image. The lower left and right panels show FDG-PET CT images; SUV max, 15.8 abnormal FDG uptake in the tumor. CT, computed tomography; FDG-PET, ${ }^{18}$ F-fluorodeoxyglucose-positron emission tomography; SUV mas, maximum standardized uptake value. 
Table II. Hematoxylin and eosin staining and immunohistochemistry results of 6 patients with primary malignant melanoma of the esophagus.

\begin{tabular}{lllcccc}
\hline & & & \multicolumn{3}{c}{ Immunohistochemistry } \\
\cline { 5 - 7 } Case no. & Sample type & H\&E & HMB45 & S-100 & melan-A & CK AE1/AE3 \\
\hline 1 & Resected specimen & Melanosis & NE & NE & NE & NE \\
2 & Resected specimen & Melanosis & + & + & NE & - \\
3 & Biopsy & Melanosis & + & NE & NE & NE \\
4 & Resected specimen & Melanosis & + & + & + & - \\
5 & Resected specimen & Melanosis & - & + & - & NE \\
6 & Biopsy & Melanosis & + & - & + & - \\
\hline
\end{tabular}

H\&E, hematoxylin amd eosin staining; HMB45, human melanin black 45; CK, cytokeratin; NE, not evaluated; +, positive; -, negative.

A

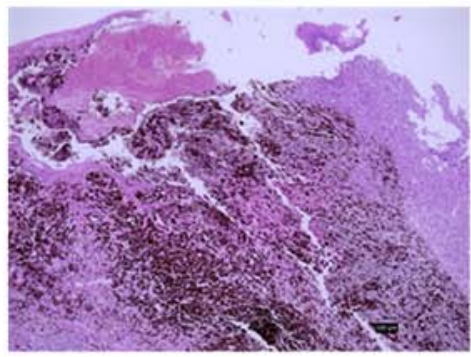

D

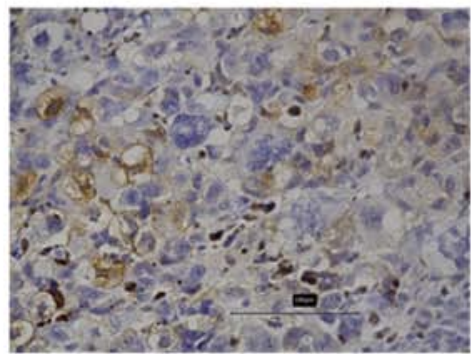

B

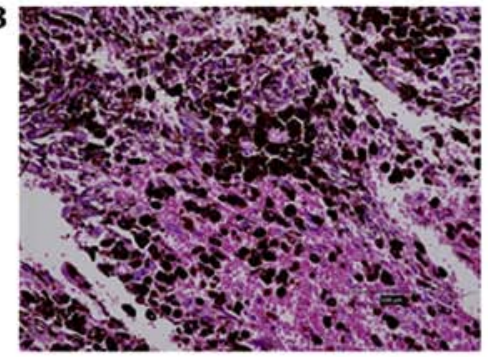

$\mathbf{E}$

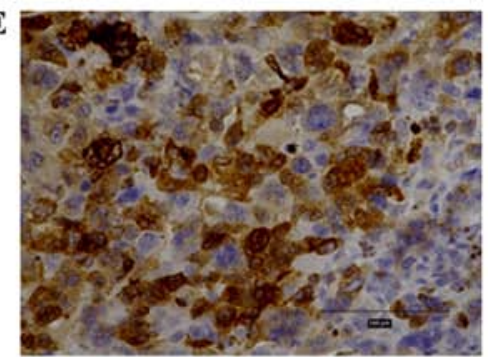

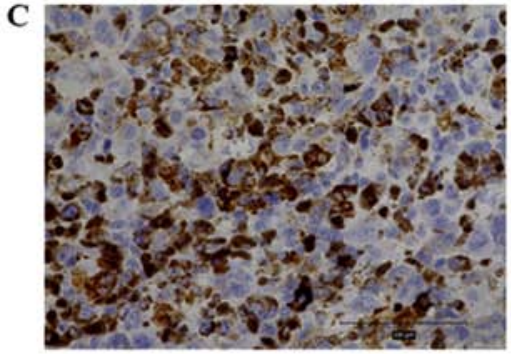

$\mathbf{F}$

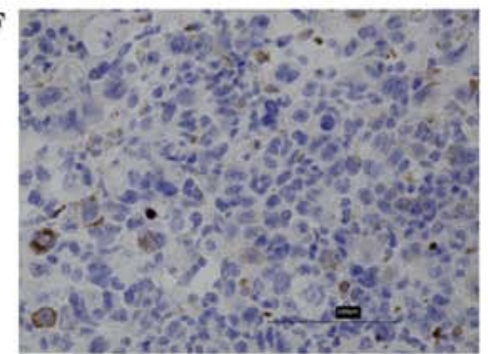

Figure 2. Representative images of $\mathrm{H} \& \mathrm{E}$ and immunohistochemical staining of primary malignant melanoma of the esophagus sections. (A) H\&E staining; magnification, x40. (B) H\&E staining; magnification, x200. (C) Positive immunohistochemical staining with human melanin black-45 antibody, magnification, x200. (D) Positive immunohistochemical staining with S-100 antibody, magnification, x200. (E) Positive immunohistochemical staining with Melan-A antibody, magnification, x200. (F) Negative immunohistochemical staining with cytokeratin AE1/AE3 antibody, magnification, x200. H\&E, hematoxylin and eosin.

antibody (Nivolumab) therapy. However, the performance status became worse over the course of treatment because of the advanced age and severe comorbidities. Therefore, the patient could no longer tolerate another cycle of anti-PD-1 antibody and thus received supportive care. The patient diagnosed with cT1bN2 PMME by endoscopic examination without biopsy (patient 1) received preoperative chemotherapy with three cycles of the DAV regimen as previously described, and the clinical response to preoperative treatment was stable disease (SD) according to the RECIST (ver.1.1) criteria. The patient misdiagnosed with cT4aN1 squamous cell carcinoma by pretherapeutic endoscopic biopsy (patient 4) received preoperative chemoradiotherapy with two cycles of the DCF regimen with concurrent irradiation $(50.4 \mathrm{~Gy} / 28 \mathrm{Fr})$, and the clinical response to chemoradiation was stable disease (SD). Four patients including those treated preoperatively received curative surgery and no postoperative complications occurred in any case except one, who developed anastomotic leakage which required treatment with drainage only.

Histopathological findings. Pathological TNM staging was pT1/2/3/4:3/0/1/0,pN0/1/2: 4/0/0, and pStage I/II/III/IV:3/1/0/0 in all cases. Histopathological examination of the two patients who received preoperative treatment revealed persistent viable cancer cells in resected specimens, and the histological response to preoperative treatment was grade 1a. Immunohistochemical and H\&E staining findings are summarized in Table II and representative findings are shown in Fig. 2. Tumor cells with melanosis were identified by $\mathrm{H} \& \mathrm{E}$ staining. Positive immunohistochemical staining for HMB45, Melan, and CK AE1/AE3 was found in the cytoplasm while S-100 staining was localized in the nucleus. H\&E staining of the primary tumor identified melanosis in all six patients with PMME. HMB-45, S-100, and melan-A positive staining was identified in $80.0 \%$ (4/5), 

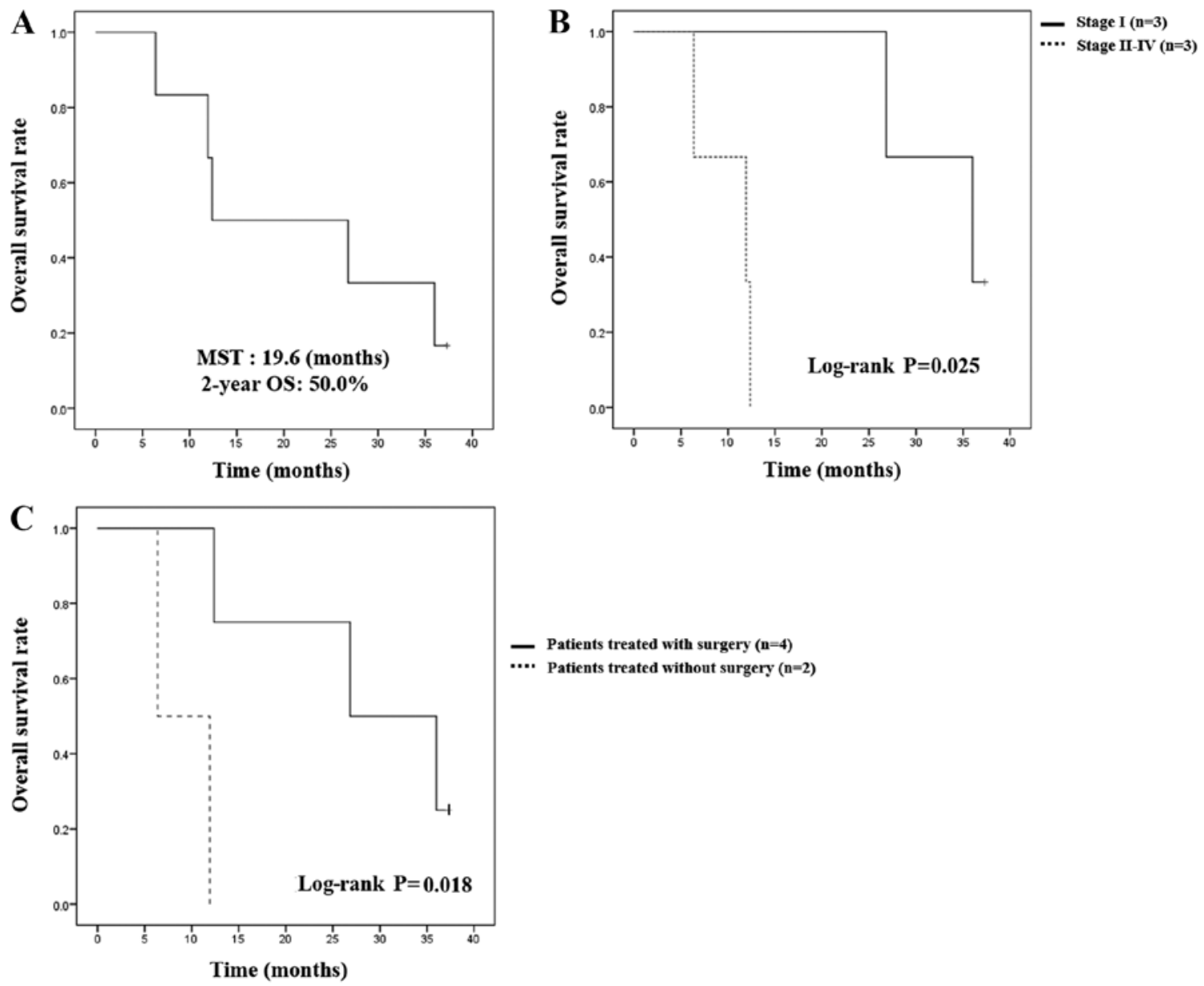

Figure 3. Kaplan-Meier OS. (A) OS in all cases. (B) OS according to Tumor-Node-Metastasis staging (pStage for resected cases, cStage for unresected cases) (C) OS according to patients treated with or without surgery. OS, overall survival; MST, median survival time.

$75.0 \%$ (3/4), and $66.7 \%$ (2/3) of patients with available immunohistochemical data, respectively. In contrast, none of the three patients with available data showed positive stating for CK AE1/AE3 (Table II).

Survival of patients with PMME. None of the four patients who received curative surgery underwent adjuvant chemotherapy and follow-up information was obtained for all of these patients. Three out of four $(75.0 \%)$ surgically-treated patients developed distant metastasis within two years of surgery, including liver in two cases (patients 1 and 4), lung in two cases (patients 2 and 4), and bone in one case (patient 4). Chemotherapy with the DAV regimen was used to treat recurrent disease in two cases while palliative therapy was performed in one case. All three patients with disease recurrence died within two years of recurrence, while only one patient (patient 5) remained alive three years after surgery without any recurrence. The median RFS was 19.3 months (range, 3.9-37.9) for patients with curative surgery, while the median OS for all patients was 19.6 months (range, 6.4-40.5) as shown in Fig. 3A. Patients with stage I disease showed significantly more favorable prognoses than those at stage II-IV (2-year OS: 100 vs. 0\%, log-rank $\mathrm{P}=0.025$ ) (Fig. 3B) and patients treated with surgery had significant better prognoses than those treated without surgery (2-year OS: 75.0 vs. $0 \%$, log-rank $\mathrm{P}=0.018)$ (Fig. 3C).

\section{Discussion}

We summarized the clinicopathological characteristics and survival of six patients with PMME. Four of five patients with endoscopic biopsies were correctly diagnosed with PMME before treatment, and most patients showed positive staining for immunomarkers including HMB-45, Melan-A, and S-100, and negative staining for the epithelium marker CK AE1/AE3. Only two of the six patients were diagnosed at an early stage and achieved long-term survival following curative surgery. Two patients who received preoperative chemo- or chemoradiotherapy showed no obvious responses while another two cases who received chemotherapy after disease recurrence had poor prognoses. Most patients also experienced hematological metastasis or recurrence even after curative surgery, underscoring the highly malignant nature of PMME and its dismal prognosis.

The prevalence of clinicopathological characteristics reported in the present study are similar to previous reports indicating a PMME prevalence rate of $0.1-0.5 \%$ of all esophageal malignancies (17-19). Patients are most commonly in their sixties or seventies with a male-to-female ratio of 2:1. Common symptoms of PMME include dysphagia, non-specific retrosternal pain, and weight loss. However, these symptoms are easily ignored and diagnosis can therefore be delayed since the clinical symptoms are unlikely to be apparent and 
specific. Of the six patients in our study, two cases diagnosed at an early stage manifested no clinical symptoms and the diagnosis was made at physical checkup. Three patients with clinical symptoms and an additional patient with no symptoms were diagnosed at advanced stages. According to the literature, PMMEs are located in the middle and lower third of the thoracic esophagus in approximately $90 \%$ of cases, probably because of the greater concentration of melanocytes in these regions. Macroscopically, tumors usually show a focally ulcerated polypoid mass mostly covered by intact squamous mucosa. These findings sometimes lead to misdiagnosis of poorly differentiated SCC with endoscopic biopsy, as seen in one of our cases. A previous study reported that biopsies were conducted in approximately $70 \%$ of patients and the diagnostic accuracy was approximately $80 \%$, while $20-50 \%$ of patients are reported to be misdiagnosed with a poorly differentiated carcinoma due to the absence of melanin granules (20). In addition, the tumor characteristics of the soft and polypoid mass may mask clinical symptoms in patients with PMME, suggesting that physicians must consider the fact that polypoid masses in the middle and lower third of the esophagus may be special types of cancer, specifically PMME. Immunohistochemical staining is a useful and reliable tool for the histological diagnosis of PMME. The majority of the resected specimens are reported to show positivity for immunomarkers including HMB-45, Melan-A, and S-100, while epithelium markers such as CK AE1/AE3 are negative, as seen in the present study (21). However, accurate PMME diagnosis before surgery appears to be difficult even with endoscopic biopsy, and tumors lacking the characteristic dark surface and microscopic melanin granules could be easily confounded with poorly differentiated carcinomas. Our study also showed that one of five patients was misdiagnosed with poorly differentiated SCC and directed to chemoradiotherapy as preoperative treatment, which was ineffective. Partial biopsy for cutaneous malignant melanoma has been contraindicated due to the risk of dissemination or metastasis, and therefore excisional biopsy should be performed for lesions suspicious for melanoma. In the past, biopsy for PMME was not conducted for this reason, as seen for one patient in our study. However, a direct correlation between biopsy for PMME and disseminated spread has not been identified, and biopsy combined with immunohistochemical staining is now widely conducted.

Multidisciplinary treatments for PMME including radical surgery, chemotherapy, chemoradiotherapy, endocrine-therapy, and immunotherapy are currently in clinical use, but PMME cases with long-term survival are rarely reported and chemotherapy and chemoradiotherapy were found to be ineffective for PMME in the present study. Immune-checkpoint inhibitors including anti-PD-1 antibody (nivolumab) and anti-CTLA-4 antibody (ipilimumab) represent a novel treatment strategy for malignant melanoma, were approved by the Food and Drug Administration (FDA) in 2014, and are available for use in PMME treatment in Japan. These agents have been reported to demonstrate a substantial clinical benefit for patients with metastatic melanoma with objective response rates of 31.0-40.0\% (22-24). In addition, several single case reports suggested that the usefulness of immunotherapy with nivolumab for PMME may be comparable to melanoma of other organs $(6,25,26)$. Additional studies with larger sample 
sizes are needed to establish the evidence for immune-checkpoint inhibitor therapy for PMME.

The literature regarding survival of PMME patients includes seven reports analyzing the prognosis of five or more patients with PMME as summarized in Table III $(3,4,27-31)$. The survival rates of PMME patients appear to be dismal, with a median overall survival of 8 to 34.5 months and 5-year overall survival rate of approximately $20 \%$ or less. Hematological and lymphatic metastases are the most common patterns of metastasis and the most common metastatic organ is liver in all cases, followed by mediastinum, mediastinal lymph nodes, lung, and brain (17). This is in agreement with the present study in which four of six patients experienced hematological metastasis or recurrence with a median survival time of 19.6 months. Patients treated with curative surgery or who had cStage I disease showed significantly better prognoses compared to those without in the survival analysis in the present study, and similar results have been reported previously.

The critical limitations of this study include the retrospective single-institution design, the greater than 20-year time period, and a sample size too small to allow for conclusive statements regarding PMME. However, since most previous reports investigating $\mathrm{PMME}$ were also single institution-based and included a much smaller number of cases than ours, the present study represents one of the largest series ever reported. Additional accumulation of evidence will be necessary for a better understanding of PMME.

In conclusion, PMME appears to be a highly malignant tumor with high metastatic potential associated with dismal survival and which may not respond to chemo-agents or radiotherapy. Diagnosis during the early stage and radical resection may be essential for long-term survival in patients with PMME. Although additional studies with a larger number of patients are necessary to validate the significance of these findings, the present report offers important information that could ultimately lead to the establishment of an optimal treatment strategy for PMME.

\section{Acknowledgements}

Not applicable.

\section{Funding}

No funding was received.

\section{Availability of data and materials}

The datasets used and/or analyzed during the current study are available from the corresponding author on reasonable request.

\section{Authors' contributions}

TH, TM, YD and MMor conceived and designed the study. TH and TM analyzed the acquired and analyzed the data, and wrote the manuscript. MY, KT, YM, TT, YKu, MMot, YKi, $\mathrm{KN}$ and EM contributed to the interpretation of data. MY, KT, YM, TT, YKu, MMot, YKim, KN, EM, YD and MMor critically revised the manuscript for important intellectual content. All authors read and approved the final manuscript.

\section{Ethics approval and consent to participate}

The present study was approved by the Institutional Review Board of Osaka University Hospital (approved project no. 18190), and it conforms to the provisions of the Declaration of Helsinki. Written informed consent was obtained from all individuals in the present study.

\section{Patient consent for publication}

Informed consent was obtained from all individuals in the present study.

\section{Competing interests}

The authors declare that they have no competing interests.

\section{References}

1. Volpin E, Sauvanet A, Couvelard A and Belghiti J: Primary malignant melanoma of the esophagus: A case report and review of the literature. Dis Esophagus 15: 244-249, 2002.

2. Iwanuma $\mathrm{Y}$, Tomita N, Amano T, Isayama F, Tsurumaru M, Hayashi T and Kajiyama Y: Current status of primary malignant melanoma of the esophagus: Clinical features, pathology, management and prognosis. J Gastroenterol 47: 21-28, 2012.

3. Gao S, Li J, Feng X, Shi S and He J: Characteristics and surgical outcomes for primary malignant melanoma of the esophagus. Sci Rep 6: 23804, 2016.

4. Yu H, Huang XY, Li Y, Xie X, Zhou JL, Zhang LJ, Fu JH and Wang X: Primary malignant melanoma of the esophagus: A study of clinical features, pathology, management and prognosis. Dis Esophagus 24: 109-113, 2011.

5. Zheng J, Mo H, Ma S and Wang Z: Clinicopathological findings of primary esophageal malignant melanoma: Report of six cases and review of literature. Int J Clin Exp Pathol 7: 7230-7235, 2014.

6. Makuuchi H, Takubo K, Yanagisawa A and Yamamoto S: Esophageal malignant melanoma: Analysis of 134 cases collected by the Japan Esophageal Society. Esophagus 12: 158-169, 2015.

7. Inadomi K, Kumagai H, Arita S, Tsuruta N, Takayoshi K, Mishima K, Ota S, Tanaka M, Okumura Y, Sagara K, et al: Bi-cy topenia possibly induced by anti-PD-1 antibody for primary malignant melanoma of the esophagus: A case report. Medicine (Baltimore) 95: e4283, 2016.

8. Sobin LH, Gospodarowicz M and Wittekind C (eds.): TNM Classification of Malignant Tumors, 7th edition. Wiley-Blackwell, Oxford, 2009.

9. Eisenhauer EA, Therasse P, Bogaerts J, Schwartz LH, Sargent D, Ford R, Dancey J, Arbuck S, Gwyther S, Mooney M, et al: New response evaluation criteria in solid tumours: Revised RECIST guideline (version 1.1). Eur J Cancer 45: 228-247, 2009.

10. Japan Esophageal Society: Japanese Classification of Esophageal Cancer, 11th edition: Part I. Esophagus 14: 1-36, 2017.

11. Makino T, Yamasaki M, Tanaka K, Masuike Y, Tatsumi M, Motoori M, Kimura Y, Hatazawa J, Mori M and Doki Y: Metabolic tumor volume change predicts long-term survival and histological response to preoperative chemotherapy in locally advanced esophageal cancer. Ann Surg, May 1, 2018 (Epub ahead of print). doi: 10.1097/SLA.0000000000002808.

12. Makino T, Miyata H, Yamasaki M, Fujiwara Y, Takiguchi S, Nakajima K, Higuchi I, Hatazawa J, Mori M and Doki Y: Utility of response evaluation to neo-adjuvant chemotherapy by (18)F-fluorodeoxyglucose-positron emission tomography in locally advanced esophageal squamous cell carcinoma. Surgery 148: 908-918, 2010.

13. Ueda E, Kishimoto S and Yasuno H: Statistical survey from 1982 to 1991 of 49 patients with malignant melanocytic tumors. J Dermatol 22: 467-474, 1995.

14. Makino T, Yamasaki M, Miyazaki Y, Wada N, Takahashi T, Kurokawa Y, Nakajima K, Takiguchi S, Mori M and Doki Y: Utility of initial induction chemotherapy with 5-fluorouracil, cisplatin, and docetaxel (DCF) for T4 esophageal cancer: A propensity score-matched analysis. Dis Esophagus 31, 2018. doi: $10.1093 /$ dote/dox 130 . 
15. Makino T, Yamasaki M, Tanaka K, Tatsumi M, Takiguchi S, Hatazawa J, Mori M and Doki Y: Importance of positron emission tomography for assessing the response of primary and metastatic lesions to induction treatments in $\mathrm{T} 4$ esophageal cancer. Surgery 162: 836-845, 2017.

16. Yamasaki M, Miyata H, Fujiwara Y, Takiguchi S, Nakajima K, Kurokawa Y, Mori M and Doki Y: Minimally invasive esophagectomy for esophageal cancer: Comparative analysis of open and hand-assisted laparoscopic abdominal lymphadenectomy with gastric conduit reconstruction. J Surg Oncol 104: 623-628, 2011.

17. Chalkiadakis G, Wihlm JM, Morand G, Weill-Bousson M and Witz JP: Primary malignant melanoma of the esophagus. Ann Thorac Surg 39: 472-475, 1985.

18. Oshiro T, Shimoji H, Matsuura F, Uchima N, Kinjo F, Nakayama T and Nishimaki T: Primary malignant melanoma of the esophagus arising from a melanotic lesion: Report of a case. Surg Today 37: 671-675, 2007.

19. Bisceglia M, Perri F, Tucci A, Tardio M, Panniello G, Vita G and Pasquinelli G: Primary malignant melanoma of the esophagus: A clinicopathologic study of a case with comprehensive literature review. Adv Anat Pathol 18: 235-252, 2011.

20. Sabanathan S, Eng J and Pradhan GN: Primary malignant melanoma of the esophagus. Am J Gastroenterol 84: 1475-1481, 1989.

21. Stranks GJ, Mathai JT and Rowe-Jones DC: Primary malignant melanoma of the oesophagus: Case report and review of surgical pathology. Gut 32: 828-830, 1991.

22. Topalian SL, Sznol M, McDermott DF, Kluger HM, Carvajal RD, Sharfman WH, Brahmer JR, Lawrence DP, Atkins MB, Powderly JD, et al: Survival, durable tumor remission, and long-term safety in patients with advanced melanoma receiving nivolumab. J Clin Oncol 32: 1020-1030, 2014.

23. Weber JS, D'Angelo SP, Minor D, Hodi FS, Gutzmer R, Neyns B, Hoeller C, Khushalani NI, Miller WH Jr, Lao CD, et al: Nivolumab versus chemotherapy in patients with advanced melanoma who progressed after anti-CTLA-4 treatment (CheckMate 037): A randomised, controlled, open-label, phase 3 trial. Lancet Oncol 16: 375-384, 2015.
24. Robert C, Long GV, Brady B, Dutriaux C, Maio M, Mortier L, Hassel JC, Rutkowski P, McNeil C, Kalinka-Warzocha E, et al: Nivolumab in previously untreated melanoma without BRAF mutation. N Engl J Med 372: 320-330, 2015.

25. Rochefort P, Roussel J, de la Fouchardière A, Sarabi M, Desseigne F, Guibert P, Cattey-Javouhey A, Mastier C, Neidhardt-Berard EM and de la Fouchardière C: Primary malignant melanoma of the esophagus, treated with immunotherapy: A case report. Immunotherapy 10: 831-835, 2018.

26. Sasaki K, Uchikado Y, Omoto I, Amatatsu M, Megumi K, Okumura $\mathrm{H}$, Maemura $\mathrm{K}$ and Natsugoe S: Multidisciplinary therapy for metastatic primary malignant melanoma of the esophagus: A case report. Mol Clin Oncol 8: 533-538, 2018

27. Lohmann CM, Hwu WJ, Iversen K, Jungbluth AA and Busam KJ: Primary malignant melanoma of the oesophagus: A clinical and pathological study with emphasis on the immunophenotype of the tumours for melanocyte differentiation markers and cancer/testis antigens. Melanoma Res 13: 595-601, 2003.

28. Li B, Lei W, Shao K, Zhang C, Chen Z, Shi S and He J: Characteristics and prognosis of primary malignant melanoma of the esophagus. Melanoma Res 17: 239-242, 2007.

29. Wang S, Tachimori Y, Hokamura N, Igaki H, Kishino T and Kushima R: Diagnosis and surgical outcomes for primary malignant melanoma of the esophagus: A single-center experience. Ann Thorac Surg 96: 1002-1006, 2013.

30. Harada K, Mine S, Yamada K, Shigaki H, Oya S, Baba H and Watanabe M: Long-term outcome of esophagectomy for primary malignant melanoma of the esophagus: A single-institute retrospective analysis. Dis Esophagus 29: 314-319, 2016.

31. Sun H, Gong L, Zhao G, Zhan H, Meng B, Yu Z and Pan Z: Clinicopathological characteristics, staging classification, and survival outcomes of primary malignant melanoma of the esophagus. J Surg Oncol 117: 588-596, 2018.

This work is licensed under a Creative Commons Attribution-NonCommercial-NoDerivatives 4.0 International (CC BY-NC-ND 4.0) License. 